\title{
When ICU Treatment Becomes Futile
}

\section{Jean-Louis Vincent ${ }^{*}$}

Department of Intensive Care, Erasme Hospital, Université libre de Bruxelles, Brussels, Belgium

${ }^{*}$ Corresponding author: Jean-Louis Vincent, Department of Intensive Care, Erasme Hospital, Brussels, Belgium, Tel: +32 25553380 ; Fax: +32 25554555 ; E-mail: jlvincen@ulb.ac.be

Rec date: Feb 26, 2014, Acc date: Jul 05, 2014, Pub date: Jul 07, 2014

Copyright: $(2014$ Vincent $\mathrm{JL}$. This is an open-access article distributed under the terms of the Creative Commons Attribution License, which permits unrestricted use, distribution, and reproduction in any medium, provided the original author and source are credited.

\begin{abstract}
Many patients admitted to the intensive care unit (ICU) have no hope of meaningful survival and receive "futile" therapy. When a patient is no longer capable of deciding (so that autonomy is no longer applicable), giving futile treatment is against the three other key ethical principles: beneficence, non-maleficence, and distributive justice. Continuing futile treatments impacts on the patient and his/her family, but also on other patients and society as a whole. In such situations, the goal of intensive care should no longer be to maximize a patient's chances of a good quality of life on discharge but rather to provide them with a dignified and comfortable death. Once a decision has been made that intervention is futile, all on-going treatment should thus be withdrawn except for comfort measures. Good and continuing communication with the family and all members of the healthcare team is an essential aspect of insuring the best possible dying process. In this article, we will explore this complex area and try to provide answers to some of the difficult issues involved, including how futility can be recognized, who should determine that further treatment is futile, and what should be done once it has been decided that further treatment is futile in a particular patient.
\end{abstract}

Keywords: ICU; Futile; Withhold; Withdraw; End-of-life

\section{Introduction}

The word 'futility' is derived from the Latin word futilis, literally meaning "pouring out easily" or "leaky" and hence considered of no use or worthless. In terms of critical care, futility is generally considered as the condition when a patient receives treatment that will have no beneficial effect in terms of quality of life or duration; there may be a physiological effect but this will not correspond to benefit for the patient. Importantly, it is the treatment that is futile and not the patient, and futility should not be considered synonymous with failure. When a patient is no longer capable of deciding (so that autonomy is no longer applicable), giving futile treatment is against the three other key ethical principles: beneficence, because it offers no benefit to the patient; against non-maleficence, because it may harm the patient; and against distributive justice, because the additional time and cost expended on providing treatment to a patient who will not survive may be better used on patients who would benefit.

But how can such a state be recognized, who should determine futility, and what should be done once it has been decided that further treatment is futile in a particular patient? We will review and explore the available literature in this field to try and provide some answers to the difficult issues involved in this complex ethical issue.

\section{Definition and Recognition of Futility}

Futile intensive care has been defined as "use of considerable resources without a reasonable hope that the patient would recover to a state of relative independence or be interactive with his or her environment" [1]. A more recent definition is "interventions that prolong life without achieving an effect that the patient can appreciate as a benefit" [2]. However, although these definitions may seem fairly straightforward at first glance, there are several areas of potential confusion/conflict that should be noted, not least in the meaning of the word "benefit". Benefit can be interpreted in terms of simply extending duration of life, but what about quality of life? Is providing organ support to sustain physiological functions really a benefit when the patient is clinically unresponsive?

In an attempt to quantify "futility", it has been suggested that treatment be considered futile when it is known to have a less than $1 \%$ chance of success [3], but this necessitates a good knowledge of all outcome data for the intervention in question for all groups of patients, data which are anyway not always available in the intensive care population. Moreover, in an interview-based study, although the proportion of surrogates who would want life-sustaining treatments continued decreased as the presented prognosis of their loved one decreased, $32 \%$ of surrogates still said they would have wanted treatment to be continued despite being told that the patient had a less than $1 \%$ chance of surviving, and $18 \%$ when the physician thought there was no chance of survival [4]. Others have suggested that scoring systems, such as the APACHE II or SAPS scores, that provide an indication of prognosis, could help determine which patients are not likely to benefit from therapy $[5,6]$; however, these scores were not designed for this purpose. For example, a young, previously healthy polytrauma victim may have a very high severity score, but surely warrants every effort to save his/her life.

Clearly the definition of futility is a complex and sensitive issue and raises many questions for which there are no easy answers. The Council on Ethical and Judicial Affairs of the American Medical Association reached the conclusion that a "fully objective and concrete definition of futility is unattainable" [7]. Without a clear definition, the next problem is who should then have the responsibility of making this rather subjective judgement call for individual patients. Some would argue that futility is purely a medical "diagnosis" and others that it is rather a moral judgement [8]. As a medical decision, clearly the physician in charge must make the final judgement. If futility is 
Page 2 of 4

considered a moral judgement, then the family or surrogate (or patient) may have a more important role to play in deciding what to call "futile". Whatever one's personal viewpoint, futility effectively refers to the inability to respond to treatment and thus is essentially a medical condition - some have suggested the term "medically inappropriate treatment" rather than "futile treatment" [9], highlighting the clinical nature of these decisions. However, all such decisions have a subjective component as different individuals will often have different opinions as to what constitutes "futility" in a particular patient [7,10]. Religious belief, cultural background, personal values, local legislation can influence one's interpretation of futility. There is, therefore, a risk that, if made unilaterally, the decision-maker's values (whether the physician or a surrogate) may be imposed on the patient $[11,12]$. Hence, decisions should be made after due consideration of all the clinical data and full consultation with all members of the healthcare team, and the family whenever possible.

Family involvement in end-of-life decisions varies considerably across the globe [13], traditionally being greater in North America than elsewhere and in northern Europe than in South America, Asia and southern Europe [14-17]. Family members should not be expected to make such decisions, especially those who do not have the medical knowledge to fully understand the complex medical issues involved. The psychological and moral burden of this process on grieving family members can increase the risks of post-traumatic stress disorder, anxiety and depression [18]. Nevertheless, in general, the trend is moving towards increased family involvement in these decisions worldwide [19-21]. However, although families should be involved in discussions and kept closely informed, the final decision should remain the responsibility of the physician-in-charge. The reasons why the decision is a medical one and why stopping or not starting treatment is the most reasonable option must be clearly explained to the family members. Indeed, healthcare providers must never feel obliged or pressured to provide futile treatment when there is medical consensus that it will be ineffective [22,23], although studies report that physicians are often influenced by family wishes [24].

\section{The Extent and Impact of "Futility" in Intensive Care}

The continuation of futile treatment in an ICU patient impacts not only on that particular patient, potentially prolonging suffering and the natural dying process, but also on family members and on other patients who could have benefited from the intensive care facilities being used by the patient receiving futile therapy. This latter concept raises the ethical principal of distributive justice and in some parts of the world (including in Europe), where the number of ICU beds is quite limited, this can be a major practical issue [25,26]. Yet, a considerable proportion of ICU patients are considered by physicians and other healthcare workers to be receiving "futile" care. In a survey of European ICU physicians, $73 \%$ of the respondents said that their ICU frequently admitted patients with no hope of survival [15]. In a survey of medical and nursing unit directors from Canadian ICUs, $87 \%$ of physicians and $95 \%$ of nurses said that futile care had been given in their ICU over the past year [27]. In a survey of 1953 ICU nurses and physicians from 82 adult ICUs in 9 European countries and Israel, $27 \%$ perceived that care was inappropriate in at least 1 patient and in $57 \%$ of these cases it was considered inappropriate because it was excessive [28]. In a recent survey of critical care specialists in 5 ICUs from one medical centre, $11 \%$ of patients were perceived by the intensivists as receiving futile treatment and $8.6 \%$ as receiving probably futile treatment [2]. These authors estimated that the cost of futile treatment in critical care was \$2.6 million over the 3-month study period. Clearly, futile interventions account for a considerable proportion of ICU interventions and are associated with high costs. So how should this problem be approached?

\section{Withholding/Withdrawing in the ICU}

The majority of deaths in the ICU are now preceded by a decision to limit treatment in some way, although the actual percentage may vary among units and across national and international borders [15,29-32]. There are various forms of withholding/withdrawing ranging from the do-not-resuscitate order in which resuscitation efforts are withheld in the case of cardiorespiratory arrest, through donot-escalate orders in which no new therapies are started but current interventions maintained, to the withdrawal of all on-going treatment (Table 1). The appropriateness of each of these decisions will depend on the individual situation. As noted earlier, decisions to withhold or withdraw futile treatments are influenced by multiple factors, including physician age and sex, religious beliefs and cultural background [33], peer pressure, and fear of litigation [15,29,34]. Importantly, it is generally accepted that there is no ethical or moral distinction between withholding and withdrawing life-sustaining treatments $[19,22,35]$. However, this is not accepted everywhere: in Israel for example, a law authorizes the use of timers on ventilators so that the respirator can stop by itself after a programmed amount of time, without a physician's intervention to withdraw the therapy $[36,37]$.

\begin{tabular}{|l|}
\hline -Do-not-resuscitate (DNR): do everything, except in case of cardiac arrest \\
\hline $\begin{array}{l}\text {-Do-not-escalate for some interventions e.g., endotracheal intubation, renal } \\
\text { replacement therapy, vasopressor therapy... }\end{array}$ \\
\hline -Do-not-escalate for anything (DNE) \\
\hline -Withdrawal of life-support \\
\hline -Increased doses of sedatives \\
\hline
\end{tabular}

Table 1: Different types of end-of-life decisions

In any case, withdrawing is seen by many as more difficult than the more "passive" withholding [13]. In some situations, withdrawing may be seen as preferable [38], allowing the patient the benefit of the doubt and a trial of therapy or "time-limited trial", sometimes referred to as "the ICU test" [39]. Importantly, if it is decided that a trial of therapy should be conducted, time limits and targets for the trial must be set in advance and, if treatment goals are not met by the allocated time, treatment should be withdrawn according to the plan [38]. Good communication with the family is particularly important in this situation to insure that the purpose of the trial is clearly understood.

Essentially, when it has been decided that treatment is futile, all therapeutic interventions already on-going should be withdrawn and no new interventions should be started. The decision, resulting from a consensus among the caregivers, should be clearly documented in the patient's notes and a plan of action determined and agreed on by the healthcare team. The family should be approached regarding the possibility of organ donation if relevant. Although much has been written about the ethical issues surrounding end-of-life care in the ICU, relatively little has been published regarding the practicalities of the process of withholding/withdrawing $[20,35,40]$. Yet, with as many as $80 \%$ of ICU deaths now associated with an end-of-life decision [41-44], information regarding how best to implement the decision to 
Page 3 of 4

withhold/withdraw is important to insure a "good" dying process for the patient. Throughout the terminal process, it must be remembered that it is treatment that is being withdrawn or withheld and not "care" $[19,20]$. Good communication among medical staff and with patients (where possible) and family members is essential [40] and all discussions, decisions, plans, and outcomes should be carefully documented $[30,35]$.

Many recommend the discontinuation of all forms of monitoring to provide as peaceful an environment as possible for the dying process [20]. However, others prefer to continue minimal monitoring so that the patient's course can be followed. In either case, there is no need to prolong the withdrawal process, as long as time has been taken to explain to family members what they can expect. Most treatments should be removed immediately, for example antibiotics, blood products, intravenous fluids and nutrition [35]. The only real reason for removing a therapy more slowly is if rapid withdrawal could cause distress or discomfort to the patient, for example, when withdrawing ventilator support, or be upsetting for the relatives. In the mechanically ventilated patient, withdrawal of ventilator support can be performed by transition from full support to T-piece or extubation over a period of 10-20 minutes [20,30], but others prefer to maintain mechanical ventilation with a progressive decrease in the respiratory settings. Adequate sedation and analgesia should be given to insure the patient remains comfortable at all times, using sufficient doses even if this speeds death $[20,35]$. In some units, including in Belgium, medication, such as analgesics/sedatives, is commonly used even if there is no apparent discomfort and the dying process may be shortened, because this can improve the quality of dying [19]. This practice is often even encouraged by family members at the bedside, who can feel that once a decision has been made the end-of-life process should not be prolonged. The complex ethical debate surrounding this approach is beyond the scope of this review. Although many patients will die soon after therapy has been withdrawn, others may remain relatively stable for a prolonged period of time. These are difficult situations for all involved, and it is important that the patient and family do not feel neglected in any way and are kept fully informed. The attending physician and nursing staff remain responsible for the well-being of their patient and the withdrawal process should be considered and conducted with the same care and concentration as any other medical procedure. Regular attendance at the bedside, updating and reassuring the relatives, answering questions, are all essential until the goal is achieved satisfactorily.

\section{Conclusion}

Continuing futile therapy is against three key ethical principles: against beneficence, because it offers no benefit to the patient; against non-maleficence, because it may harm the patient; and against distributive justice, because the additional time and cost expended could be better used on patients who would benefit. It is, therefore, important that futility of the treatment is acknowledged so that a decision to withhold/withdraw on-going support can be taken. Patients and families should be involved in these discussions, but as with other medical interventions on the ICU, withholding/ withdrawing therapy is ultimately a medical judgment. Once the decision has been made, every effort should be taken to insure a peaceful and dignified death.

\section{References}

1. Sibbald R, Downar J, Hawryluck L (2007) Perceptions of "futile care" among caregivers in intensive care units. CMAJ 177: 1201-1208.

2. Huynh TN, Kleerup EC, Wiley JF, Savitsky TD, Guse D, et al. (2013) The frequency and cost of treatment perceived to be futile in critical care. JAMA Intern Med 173: 1887-1894.

3. Schneiderman LJ, Jecker NS, Jonsen AR (1990) Medical futility: its meaning and ethical implications. Ann Intern Med 112: 949-954.

4. Zier LS, Burack JH, Micco G, Chipman AK, Frank JA, et al. (2009) Surrogate decision makers' responses to physicians' predictions of medical futility. Chest 136: 110-117.

5. Luce JM, Wachter RM (1994) The ethical appropriateness of using prognostic scoring systems in clinical management. Crit Care Clin 10: 229-241.

6. Luce JM (1995) Physicians do not have a responsibility to provide futile or unreasonable care if a patient or family insists. Crit Care Med 23: 760-766.

7. (1999) Medical futility in end-of-life care: report of the Council on Ethical and Judicial Affairs. JAMA 281: 937-941.

8. Helft PR, Siegler M, Lantos J (2000) The rise and fall of the futility movement. N Engl J Med 343: 293-296.

9. Wilkinson DJ, Savulescu J (2011) Knowing when to stop: futility in the ICU. Curr Opin Anaesthesiol 24: 160-165.

10. Frick S, Uehlinger DE, Zuercher Zenklusen RM (2003): Medical futility: predicting outcome of intensive care unit patients by nurses and doctors--a prospective comparative study. Crit Care Med 31: 456-461.

11. Rubin SB (2007): If we think it's futile, can't we just say no? HEC Forum 19:45-65.

12. Lantos JD, Singer PA, Walker RM, Gramelspacher GP, Shapiro GR, et al. (1989) The illusion of futility in clinical practice. Am J Med 87:81-84.

13. Vincent JL (2004) Ethical principles in end-of-life decisions in different European countries. Swiss Med Wkly 134: 65-68.

14. Carlet J, Thijs LG, Antonelli M, Cassell J, Cox P, et al. (2004) Challenges in end-of-life care in the ICU. Statement of the 5th International Consensus Conference in Critical Care: Brussels, Belgium, April 2003. Intensive Care Med 30: 770-784.

15. Vincent JL (1999) Forgoing life support in western European intensive care units: the results of an ethical questionnaire. Crit Care Med 27: 1626-1633.

16. Cohen S, Sprung C, Sjokvist P, Lippert A, Ricou B, et al. (2005) Communication of end-of-life decisions in European intensive care units. Intensive Care Med 31: 1215-1221.

17. Moselli NM, Debernardi F, Piovano F (2006) Forgoing life sustaining treatments: differences and similarities between North America and Europe. Acta Anaesthesiol Scand 50: 1177-1186.

18. Azoulay E, Pochard F, Kentish-Barnes N, Chevret S, Aboab J, et al. (2005) Risk of post-traumatic stress symptoms in family members of intensive care unit patients. Am J Respir Crit Care Med 171: 987-994.

19. Vincent JL, Schetz M, De Waele JJ, de Clety SC, Michaux I, et al. (2014) "Piece" of mind: end of life in the intensive care unit statement of the Belgian Society of Intensive Care Medicine. J Crit Care 29: 174-175.

20. Rubenfeld GD (2004) Principles and practice of withdrawing lifesustaining treatments. Crit Care Clin 20: 435-451.

21. Johnson SK, Bautista CA, Hong SY, Weissfeld L, White DB (2011) An empirical study of surrogates' preferred level of control over value-laden life support decisions in intensive care units. Am J Respir Crit Care Med 183: 915-921.

22. Morrison LJ, Kierzek G, Diekema DS, Sayre MR, Silvers SM, et al. (2010) Part 3: ethics: 2010 American Heart Association Guidelines for Cardiopulmonary Resuscitation and Emergency Cardiovascular Care. Circulation 122: S665-S675.

23. Curtis JR, Tonelli MR (2011) Shared decision-making in the ICU: value, challenges, and limitations. Am J Respir Crit Care Med 183: 840-841. 
24. Kubler A, Adamik B, Lipinska-Gediga M, Kedziora J, Strozecki L (2011) End-of-life attitudes of intensive care physicians in Poland: results of a national survey. Intensive Care Med 37: 1290-1296.

25. Vincent JL, Marshall JC, Namendys-Silva SA, Francois B, MartinLoeches I, et al. (2014) Assessment of the worldwide burden of critical illness: the Intensive Care Over Nations (ICON) audit. Lancet Respir Med 2: 380-386.

26. Ball CG, Navsaria P, Kirkpatrick AW, Vercler C, Dixon E, et al. (2010) The impact of country and culture on end-of-life care for injured patients: results from an international survey. J Trauma 69: 1323-1333.

27. Palda VA, Bowman KW, McLean RF, Chapman MG (2005) "Futile" care do we provide it? Why? A semistructured, Canada-wide survey of intensive care unit doctors and nurses. J Crit Care 20: 207-213.

28. Piers RD, Azoulay E, Ricou B, Dekeyser GF, Decruyenaere J, et al. (2011) Perceptions of appropriateness of care among European and Israeli intensive care unit nurses and physicians. JAMA 306: 2694-2703.

29. Yaguchi A, Truog RD, Curtis JR, Luce JM, Levy MM, et al. (2005) International differences in end-of-life attitudes in the intensive care unit: results of a survey. Arch Intern Med 165: 1970-1975.

30. Curtis JR, Vincent JL (2010) Ethics and end-of-life care for adults in the intensive care unit. Lancet 376: 1347-1353.

31. Sprung CL, Cohen SL, Sjokvist P, Baras M, Bulow HH, et al. (2003) Endof-life practices in European intensive care units: the Ethicus Study. JAMA 290: 790-797.

32. Bertolini G, Boffelli S, Malacarne P, Peta M, Marchesi M, et al. (2010) End-of-life decision-making and quality of ICU performance: an observational study in 84 Italian units. Intensive Care Med 36: 1495-1504.

33. Sprung CL, Maia P, Bulow HH, Ricou B, Armaganidis A, et al. (2007) The importance of religious affiliation and culture on end-of-life decisions in European intensive care units. Intensive Care Med 33: 1732-1739.
34. Vincent JL (2001) Cultural differences in end-of-life care. Crit Care Med 29: N52-N55.

35. Truog RD, Campbell ML, Curtis JR, Haas CE, Luce JM, et al. (2008) Recommendations for end-of-life care in the intensive care unit: a consensus statement by the American College of Critical Care Medicine. Crit Care Med 36: 953-963.

36. Ravitsky V (2005) Timers on ventilators. BMJ 330: 415-417.

37. Steinberg A, Sprung CL (2007) The dying patient act, 2005: Israeli innovative legislation. Isr Med Assoc J 9: 550-552.

38. Vincent JL (2005) Withdrawing may be preferable to withholding. Crit Care 9: 226-229.

39. Quill TE, Holloway R (2011) Time-limited trials near the end of life. JAMA 306: 1483-1484.

40. Cook D, Rocker G (2014) Dying with dignity in the intensive care unit. N Engl J Med 370: 2506-2514.

41. Brieva JL, Cooray P, Rowley M (2009) Withholding and withdrawal of life-sustaining therapies in intensive care: an Australian experience. Crit Care Resusc 11: 266-268.

42. Hoel H, Skjaker SA, Haagensen R, Stavem K (2014): Decisions to withhold or withdraw life-sustaining treatment in a Norwegian intensive care unit. Acta Anaesthesiol Scand (in press).

43. Jensen HI, Ammentorp J, Ording H (2011) Withholding or withdrawing therapy in Danish regional ICUs: frequency, patient characteristics and decision process. Acta Anaesthesiol Scand 55: 344-351.

44. Spronk PE, Kuiper AV, Rommes JH, Korevaar JC, Schultz MJ (2009) The practice of and documentation on withholding and withdrawing life support: a retrospective study in two Dutch intensive care units. Anesth Analg; 109: 841-846. 